Sādhanā Vol. 40, Part 1, February 2015, pp. 235-248. (C) Indian Academy of Sciences

\title{
Interfacial stresses in strengthened beam with shear cohesive zone model
}

\author{
ZERGUA ABDESSELAM
}

Department of Civil Engineering, University of Constantine 1, Constantine, Algeria e-mail: zergua.abdesselam@umc.edu.dz

MS received 24 April 2014; revised 14 July 2014; accepted 12 September 2014

\begin{abstract}
The failure of strengthened beams with fibre-reinforced polymer (FRP) materials is due to high stress concentration of FRP-concrete interface. Understanding the cause and mechanism of the debonding of the FRP plate and the prediction of the stress distribution at the concrete-FRP interface are important for more effective strengthening technique. This paper presents an analytical solution, based on Smith and Teng's equations, for interfacial shear and normal stresses in reinforced concrete (RC) beams strengthened with a fibre reinforced polymer (FRP) plate. However, the shear stress-strain relationship is considered to be bilinear curve. The effects of the shear deformations are calculated in an RC beam, an adhesive layer, and an FRP plate. The results of parametric study are compared with those of Smith and Teng. They confirm the accuracy of the proposed approach in predicting both interfacial shear and normal stresses.
\end{abstract}

Keywords. Strengthened beam; interfacial stresses; cohesive zone; shear deformation.

\section{Introduction}

The FRP plates can be either externally bonded, (Lorenzis et al 2001), or near-surface mounted onto the RC structures through structural adhesives, (Hassan \& Rizkalla 2003; Lorenzis \& Teng 2006). Several studies have been carried out to predict the interfacial stresses of RC beams strengthened with an FRP plate, (Ahmad et al 2001; Maalej \& Leong 2005; Smith \& Teng 2001; Teng et al 2003; Yang et al 2004). In general, it has been shown that the interface between the FRP and concrete plays a critical role in the strengthening method. However, failure of the FRP and interface debonding can lead to premature failure of the strengthened RC beams. FRPconcrete interface debonding can be generally classified into two main types (Teng et al 2003): plate end debonding and intermediate crack-induced debonding.

Several cohesive zone model (CZM) have been developed for different materials. Needleman (1987) first proposed a polynomial law to describe the process of nucleation in metal. Xu \& Needleman (1994) developed the exponential law to study the void nucleation at the 
interface of particle and matrix material. Tvergaard \& Hutchinson (1993) used a trapezoidal traction-separation model to evaluate the crack growth resistance in elastic- plastic materials. Ortiz \& Suresh (1993) adopted a linear cohesive law for intergranular fracture behaviour. Camacho \& Ortiz (1996) employed a linearly decreasing cohesive fracture model, to propagate cracks during impact damage in brittle materials. Geubelle \& Baylor (1998) proposed a bilinear CZM to simulate delamination of thin composite plates subjected to low-velocity impact. It has been shown in experiments (Wu \& Yin 2003; Nakaba et al 2001) that a bilinear bond-slip relationship is a good approximation for the FRP-concrete interface behaviour. A number of modifications of this bi-linear law have also been proposed for the FRP-concrete interface (Yuan et al 2004; Fallah et al 2008). Wang (2007) used a nonlinear bond-slip model to simulate the shear stress-separation law of the FRP-concrete interface. Closed-form solutions of interfacial stresses, FRP stress were obtained for a typical single-lap specimen for the whole debonding process. Freddi \& Savoia (2008) studied the debonding of FRP plates making use of boundary integral equations. Mode II cohesive crack model is adopted for the interface, whereas linear elasticity is used for the two materials outside the process zone. Ouyang \& Wan (2008) derived a novel analytical solution of debonding process under peel loading for FRP-concrete bond joint with simplified bi-linear separation-stress law based on the cohesive zone model. Lorenzis \& Zavarise (2009) presented a simple analytical solution for the interfacial stresses between a beam under bending and a thin plate using the $\mathrm{CZ}$ modelling approach where only the interfacial shear stresses are considered. A closed-form expression for the plate end debonding load has been then derived. Cornetti \& Carpinteri (2011) showed that analytical solutions can be achieved also assuming an exponential decaying softening law. Accordingly, the expressions for the interfacial shear stress distribution and the load displacement response have been derived for the different loading stages. However, limited studies have been conducted on cohesive zone modelling of interfacial stresses in plated beams (Lorenzis \& Zavarise 2009).

In this paper, an analytical solution is proposed to evaluate the shear and normal stresses at the interface of simply supported reinforced concrete beam strengthened with a bonded FRP plate. Unlike Smith \& Teng's (2001) approach, the interface layer between FRP and concrete is modelled as a bi-linear bond-slip law.

\section{Basic assumption and analytical analysis}

Figure 1 shows a simple supported concrete beam subjected to a distributed transverse load $q$. The concrete beam is strengthened by means of partially bonding FRP plate at the bottom of the beam. Let ' $L$ ' denote the length of the concrete beam, ' $a$ ' denote the distance between the end of FRP plate and the support and $l_{f}$ the length of FRP plate. The width of FRP plate is the same as that of the concrete beam. The thickness of the adhesive layer is $t_{a}$. The interfacial shear stresses $\tau(x)$ and the normal stresses $\sigma(x)$ are shown with their signs in figure 2 .

The main assumptions adopted in this analysis are as follows. All materials (concrete beam, FRP plate and adhesive layer) are linear elastic solids. The interfacial layer between the concrete beam and FRP plate is perfect. The effects of transverse shear stress and shear strain in the RC beam, the FRP plate and the adhesive layer are considered. Stresses in the adhesive layer remain constant through the thickness. 
q

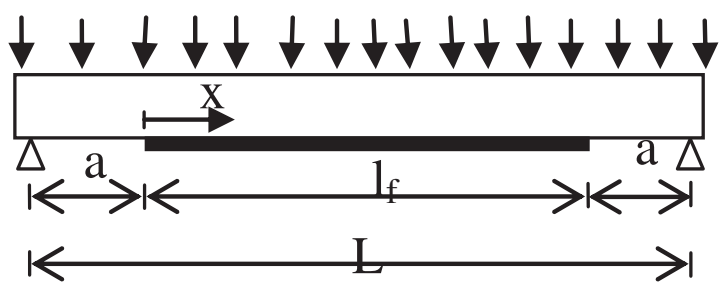

b

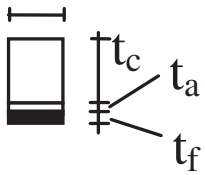

Figure 1. The strengthened beam.

\section{Shear cohesive zone model}

The cohesive zone model describes the relationships between the interfacial stresses and the relative displacements along the interface. The bi-linear bond-slip law (figure 3) by referring to the experiments reported by Nakaba et al (2001) and Wu \& Yin (2003) is adopted to model the interface layer between FRP and concrete. Three segments of this law are defined. Linear elastic state, where the interfacial shear stress increases linearly to $\tau_{\mathrm{f}}$ when the interface slip attains $\delta_{1}$; linear softening state, where the interfacial shear stress decreases linearly with the interface slip; and debonding state where the interfacial shear stress vanishes when the interface slip attains its maximum value $\delta_{\mathrm{f}}$, failure then occur by debonding of the FRP plate from the concrete beam.

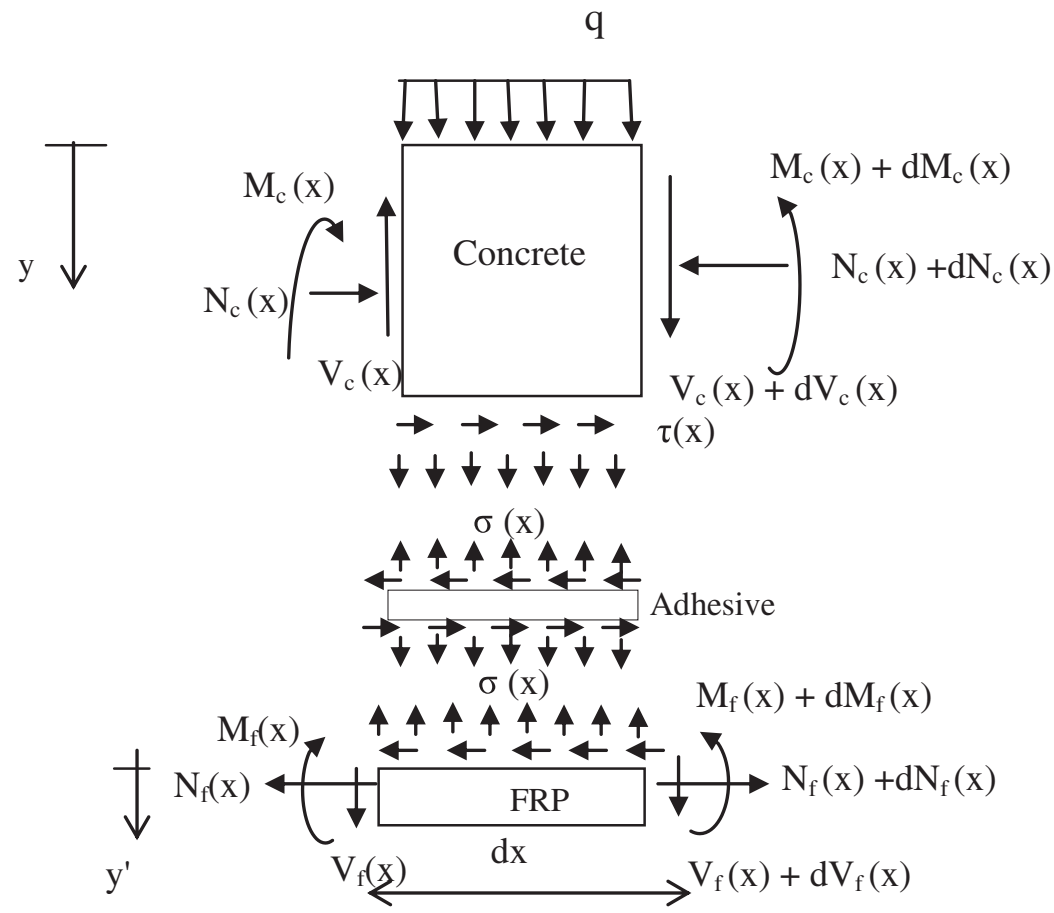

Figure 2. Forces in an infinitesimal element of a strengthened beam. 


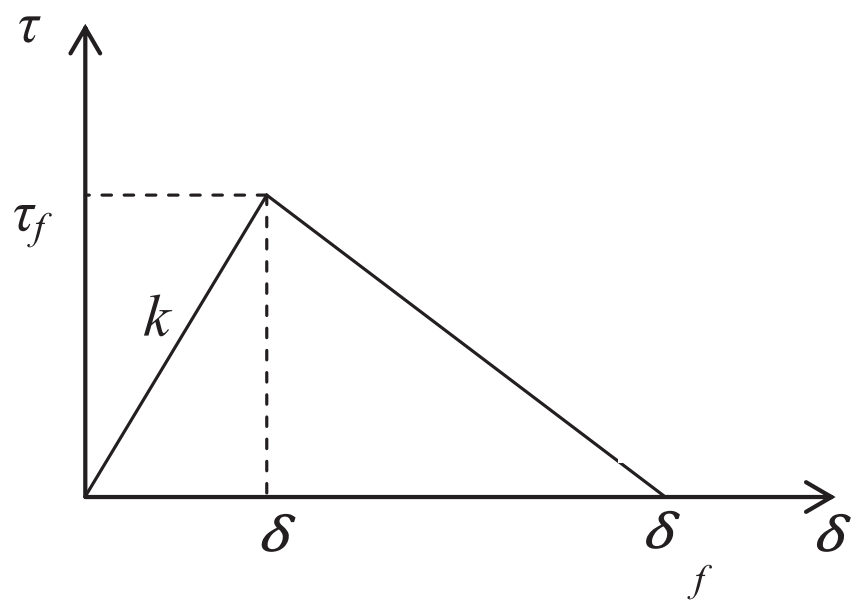

Figure 3. Bond slip law.

The interface bond-slip law, shown in figure 3, is described by the following mathematical expressions:

$$
\tau(x)=\left\{\begin{array}{cc}
\frac{\tau_{f}}{\delta_{1}} \delta & 0 \leq \delta \leq \delta_{1} \\
\frac{\delta_{f}-\delta}{\delta_{f}-\delta_{1}} \tau_{f} & \delta_{1} \leq \delta \leq \delta_{f} . \\
0 & \delta>\delta_{f}
\end{array}\right.
$$

\section{Adhesive shear stress}

Considering the infinitesimal element of a strengthened beam shown in figure 3 . The horizontal equilibrium conditions may be written as

$$
\begin{gathered}
d N_{c}(x)-b \cdot d x \cdot \tau(x)=0, \\
d N_{f}(x)-b \cdot d x \cdot \tau(x)=0, \\
\frac{d N_{c}(x)}{d x}=\frac{d N_{f}(x)}{d x}=b \tau(x) .
\end{gathered}
$$

Thus

$$
N_{c}(x)=N_{f}(x)=N(x)=b \int_{0}^{x} \tau(x) d x .
$$

The curvature of the beam and the FRP layer are supposed to be equal. Then the relationship between the moments in the two adherents can be written as follows (Smith \& Teng 2001)

$$
\begin{gathered}
\frac{d^{2} w_{c}(x)}{d x^{2}}=-\frac{M_{c}(x)}{E_{c} I_{c}}, \\
\frac{d^{2} w_{f}(x)}{d x^{2}}=-\frac{M_{f}(x)}{E_{f} I_{f}},
\end{gathered}
$$




$$
\frac{M_{c}(x)}{E_{c} I_{c}}=\frac{M_{f}(x)}{E_{f} I_{f}} \Rightarrow \quad M_{c}(x)=R M_{f}(x),
$$

with

$$
R=\frac{E_{c} I_{c}}{E_{f} I_{f}} .
$$

The equilibrium of moments acting on the infinitesimal element gives:

$$
M_{T}(x)=M_{c}(x)+M_{f}(x)+N(x)\left[y_{1}+\frac{t_{f}}{2}+t_{a}\right],
$$

where $M_{T}(x)$ is the total applied moment.

The first derivative of the bending moment in each adherent gives:

$$
\begin{aligned}
& \frac{d M_{c}(x)}{d x}=\frac{R}{R+1}\left[V_{T}(x)-b \tau(x)\left(y_{1}+\frac{t_{f}}{2}+t_{a}\right)\right], \\
& \frac{d M_{f}(x)}{d x}=\frac{1}{R+1}\left[V_{T}(x)-b \tau(x)\left(y_{1}+\frac{t_{f}}{2}+t_{a}\right)\right] .
\end{aligned}
$$

The strains at the bottom of beam element and the top of FRP plate are given by the following equations

$$
\begin{gathered}
\frac{\partial u_{c}}{\partial x}=\varepsilon_{c}(x)=\frac{y_{1}}{E_{c} I_{c}} M_{c}(x)-\frac{N_{c}(x)}{E_{c} A_{c}}+\frac{t_{c} d \tau(x)}{3 G_{c} d x}, \\
\frac{\partial u_{f}}{\partial x}=\varepsilon_{f}(x)=-\frac{y_{2}}{E_{f} I_{f}} M_{f}(x)+\frac{N_{c}(x)}{E_{f} A_{f}}-\frac{t_{f} d \tau(x)}{3 G_{f} d x} .
\end{gathered}
$$

The subscripts $\mathrm{c}$ and $f$ denote concrete and FRP materials, respectively. $M_{i}, N_{i}, E_{i}, A_{i}, t_{i}, G_{i}$ denote respectively the bending moment, the axial force, the elastic modulus, the cross-sectional area of concrete beam, the cross-sectional area, the thickness and the shear modulus of each adherent $\mathrm{i}$.

$y_{1}$ and $y_{2}$ : distances from the bottom of concrete beam to its centroid and of the centroid of the FRP plate from its upper surface, respectively.

\subsection{Linear elastic stage}

At this stage, the first branch of the cohesive law is used.

$$
\begin{array}{r}
\tau(x)=K . \delta, \\
\delta=u_{f}(x)-u_{c}(x),
\end{array}
$$

where: $u_{c}$ and $u_{f}$ are respectively the horizontal displacement at the bottom of the concrete beam and the horizontal displacement at the top of the externally bonded FRP plate.

By differentiating Eq. (15) with respect to $x$ we obtain:

$$
\frac{d \tau(x)}{d x}=k\left(\frac{\partial u_{f}(x)}{\partial x}-\frac{\partial u_{c}(x)}{\partial x}\right) .
$$


By differentiating Eqs. (13) and (14), the relative displacements between the FRP plate and the concrete beam and by including shear deformation will be given by the following equations

$$
\begin{gathered}
\frac{d^{2} u_{c}}{d x^{2}}=\frac{y_{1}}{E_{c} I_{c}} \frac{d M_{c}(x)}{d x}-\frac{1}{E_{c} A_{c}} \frac{d N_{c}(x)}{d x}+\frac{t_{c}}{3 G_{c}} \frac{d^{2} \tau(x)}{d x^{2}}, \\
\frac{d^{2} u_{f}}{d x^{2}}=-\frac{y_{2}}{E_{f} I_{f}} \frac{d M_{f}(x)}{d x}+\frac{1}{E_{f} A_{f}} \frac{d N_{f}(x)}{d x}-\frac{t_{f}}{3 G_{f}} \frac{d^{2} \tau(x)}{d x^{2}} .
\end{gathered}
$$

By differentiating twice Eq. (15) with respect to $\mathrm{x}$ we obtain:

$$
\frac{d \tau^{2}(x)}{d x^{2}}=k\left[\frac{d^{2} u_{f}(x)}{d x^{2}}-\frac{d^{2} u_{c}(x)}{d x^{2}}\right] .
$$

Substituting Eqs. (17) and (18) into Eq. (19) and by using Eqs. (5), (8) and (9) we obtain the following differential equation for the shear stress distribution in the elastic zone:

$$
\frac{d \tau^{2}(x)}{d x^{2}}+\lambda^{2} \tau(x)+\eta \quad V_{T}(x)=0,
$$

in which

$$
\begin{gathered}
\lambda^{2}=\left[\frac{\left(y_{1}+y_{2}\right)\left(y_{1}+y_{2}+t_{a}\right)}{E_{c} I_{c}+E_{f} I_{f}}+\frac{1}{E_{c} A_{c}}+\frac{1}{E_{f} A_{f}}\right] \frac{b}{\psi}, \\
\psi=\left[\frac{1}{k}+\frac{t_{f}}{3 G}+\frac{t_{a}}{3 G}\right] \quad \eta=\left[\frac{y_{1}+y_{2}}{E_{c} I_{c}+E_{f} I_{f}}\right] \frac{1}{\psi} .
\end{gathered}
$$

The general solution of the differential Eq. (20) is given by

$$
\tau(x)=C_{1} \cosh (\lambda x)+C_{2} \sinh (\lambda x)+C_{3} .
$$

The constants $C_{1}, C_{2}$ and $C_{3}$ are determined by considering the following boundary conditions: The shear stress is zero at $\mathrm{x}=l / 2$ and the axial force is zero, at $x=0$.

\subsection{Linear softening stage}

As the load increases, the interfacial shear stress reaches its maximum value at $x=0$. Then the linear softening starts at the end of the FRP plate. In this stage, the second branch of the cohesive law is used. The interfacial stress distribution in this zone is given as

$$
\tau(x)=\frac{\delta_{f}-\delta}{\delta_{f}-\delta_{1}} \tau_{f},
$$

Using

$$
\frac{d \tau^{2}(x)}{d x^{2}}=k\left[\frac{d^{2} u_{f}(x)}{d x^{2}}-\frac{d^{2} u_{c}(x)}{d x^{2}}\right] .
$$


From the above relation, the differential equation for the shear stress distribution in the linear softening zone is given by

$$
\frac{d \tau^{2}(x)}{d x^{2}}+\lambda_{1}^{2} \tau(x)+\eta_{1} \quad V_{T}(x)=0
$$

In which

$$
\begin{gathered}
\lambda_{1}^{2}=k_{2} b\left(y_{1}+t_{a}+\frac{t_{f}}{2}\right) \lambda_{2} ; \quad \lambda_{2}=\frac{\lambda_{3}}{\lambda_{4}} ; \quad \lambda_{3}=\frac{1}{E_{c} A_{c}}-\frac{1}{E_{f} A_{f}}+\frac{y_{1}+y_{2}}{E_{c} I_{c}+E_{f} I_{f}} \\
\lambda_{4}=\frac{3 G_{1} G_{2}-K_{2}\left(t_{1} G_{2}-t_{2} G_{1}\right)}{G_{1} G_{2}} \quad \eta_{1}=\frac{-k_{2} \frac{\left(y_{1}+y_{2}\right)}{E_{c} I_{c}+E_{f} I_{f}}}{\lambda_{4}} \omega=-\frac{k_{1}}{\lambda_{4}} .
\end{gathered}
$$

The general solution of the differential Eq. (22) is given by

$$
\tau(x)=C_{4} \cosh \left(\lambda_{1} x\right)+C_{5} \sinh \left(\lambda_{1} x\right)+C_{6} .
$$

The constants $C_{4}, C_{5}$ and $C_{6}$ are determined by considering the following boundary conditions: (a) the shear stress is zero at the symmetry axis, $x=l / 2$; (b) at the plate end $x=0$, the axial force is zero $N 1=N 2=0$, for both beam and plate.

\section{Adhesive normal stresses}

The normal stress in the adhesive layer is expressed as follows

$$
\sigma_{n}(x)=\varepsilon_{y} E_{a} .
$$

The strain in the adhesive layer is given by

$$
\varepsilon_{y}=\frac{\partial w(x, y)}{\partial y} \approx \frac{w_{f}(x)-w_{c}(x)}{t_{a}},
$$

where $w_{c}(x)$ and $w_{f}(x)$ are the vertical displacements of the beam (concrete) and the FRP layer, respectively.

Eq. (24) can then be written as

$$
\sigma_{n}(x)=\frac{E_{a}}{t_{a}}\left[w_{f}(x)-w_{c}(x)\right]
$$

By differentiating twice Eq. (26) with respect to x, we obtain:

$$
\frac{d^{2} \sigma_{n}(x)}{d x^{2}}=\frac{E_{a}}{t_{a}}\left[\frac{d^{2} w_{f}(x)}{d x^{2}}-\frac{d^{2} w_{c}(x)}{d x^{2}}\right] .
$$


The moment-curvature relationship for the two adherents is expressed as follows

$$
\begin{gathered}
\frac{d^{2} w_{c}(x)}{d x^{2}}=\frac{M_{c}(x)}{E_{c} I_{c}}+\frac{d \tau(x)}{3 G_{c} d x}, \\
\frac{d^{2} w_{f}(x)}{d x^{2}}=-\frac{M_{f}(x)}{E_{f} I_{f}}-\frac{d \tau(x)}{3 G_{f} d x} .
\end{gathered}
$$

The moment equilibrium of adherents 1 and 2 gives:

Adherent 1:

$$
\frac{d M_{c}(x)}{d x}=V_{c}(x)-b_{f} y_{1} \tau(x) \text { and } \frac{d V_{c}(x)}{d x}=-b_{f} \sigma(x)-q
$$

Adherent 2:

$$
\frac{d M_{f}(x)}{d x}=V_{f}(x)-b_{f} \frac{t_{f}}{2} \tau(x) \text { and } \frac{d V_{f}(x)}{d x}=b_{f} \sigma(x),
$$

where $q$ is the uniformly distributed load.

By differentiating Eq. (27) and using Eqs. (30) and (31) we obtain:

$$
\frac{d^{4} \sigma_{n}(x)}{d x}+\frac{E_{s} \cdot b}{t_{a}}\left(\frac{1}{E_{1} I_{1}}+\frac{1}{E_{f} I_{f}}\right) \sigma_{n}(x)+\frac{E_{s} \cdot b}{t_{a}}\left(\frac{y_{1}}{E_{1} I_{1}}-\frac{y_{2}}{E_{f} I_{f}}\right) \frac{\partial \tau(x)}{\partial x_{f}}+\frac{q E_{a}}{t_{a} E_{c} I_{c}}=0 .
$$

The solution of Eq. (32) gives the interfacial normal stress as:

$$
\sigma_{n}(x)=e^{-\beta x}\left[C_{4} \cos (\beta x)+C_{5} \sin (\beta x)\right]+\left[C_{6} \cos (\beta x)+C_{7} \sin (\beta x)\right]-\eta_{2} \frac{\partial \tau(x)}{\partial x_{f}}-\eta_{3} q .
$$

The constants are determined by considering the boundary conditions mentioned above.

\section{Parametric study}

Before proceeding with the parametric study, it is necessary to assess the accuracy of the proposed solution. For this purpose, interfacial shear and normal stresses of a strengthened beam obtained from the proposed method were compared to those obtained by the method developed by Smith \& Teng (2001). The strengthened beam is simply supported with a span of $300 \mathrm{~mm}$ and is subjected to a uniformly distributed load $\mathrm{w}=50 \mathrm{KN} / \mathrm{m}$. The reinforced concrete beam has a rectangular cross section of width $b=200 \mathrm{~mm}$ and height $\mathrm{h}=300 \mathrm{~mm}$. The adhesive layer and the FRP, of same width as the reinforced concrete beam, have a thickness of 2.4 and $4 \mathrm{~mm}$ respectively. The elastic properties for the different materials adopted in this study are given in table 1.

Figures 4 and 5 show the interfacial shear and normal stresses vs. distance from the end plate. From these two figures, it is observed that there is a good correlation between the two methods.

Table 1. Material properties of the analysed beam.

\begin{tabular}{lcc}
\hline Component & $\mathrm{E}(\mathrm{GPa})$ & $\mathrm{G}(\mathrm{GPa})$ \\
\hline RC beam & 30 & 14 \\
adhesive layer & 2 & 1.2 \\
FRP & 50 & 4.5 \\
\hline
\end{tabular}




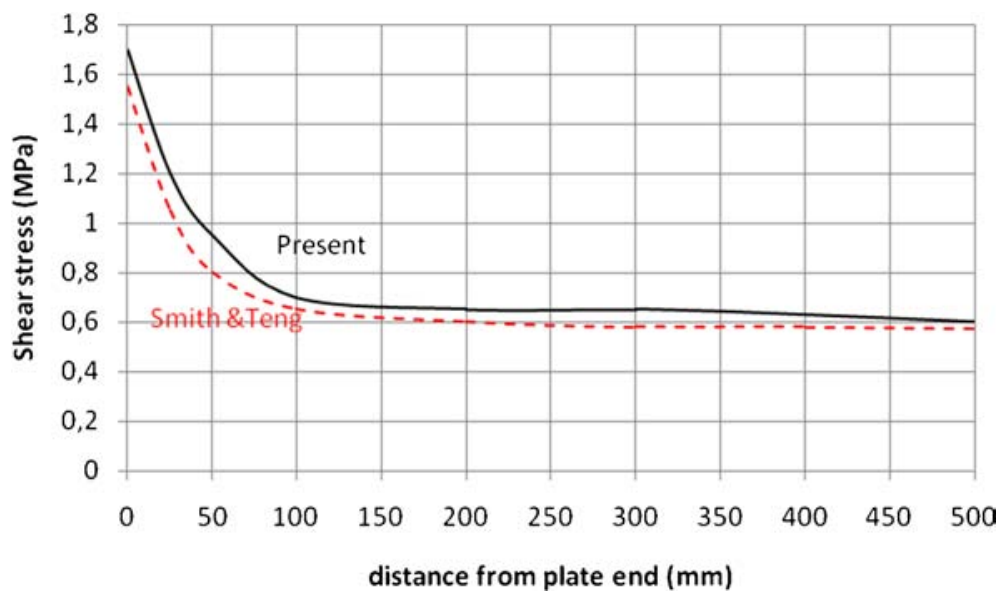

Figure 4. Comparison between the proposed solution and that of Smith and Teng for shear stresses (2001).

It can also be observed that the interfacial stresses are significant near the end of the plate. They decrease rapidly to become negligible starting from the first third of the plate.

Once the proposed analytical method was validated, parametric study was performed on beams with different configurations. The variable parameters considered in this study are thickness and elastic modulus of both adhesive layer and FRP plate.

\subsection{Effect of the adhesive layer thickness}

The effects of the adhesive layer thickness on interfacial shear and normal stresses are shown in figures 6 and 7. The maximum interfacial normal and shear stresses occurs near the end of the FRP plate. It can be seen that reducing the thickness of the adhesive layer leads to an increase

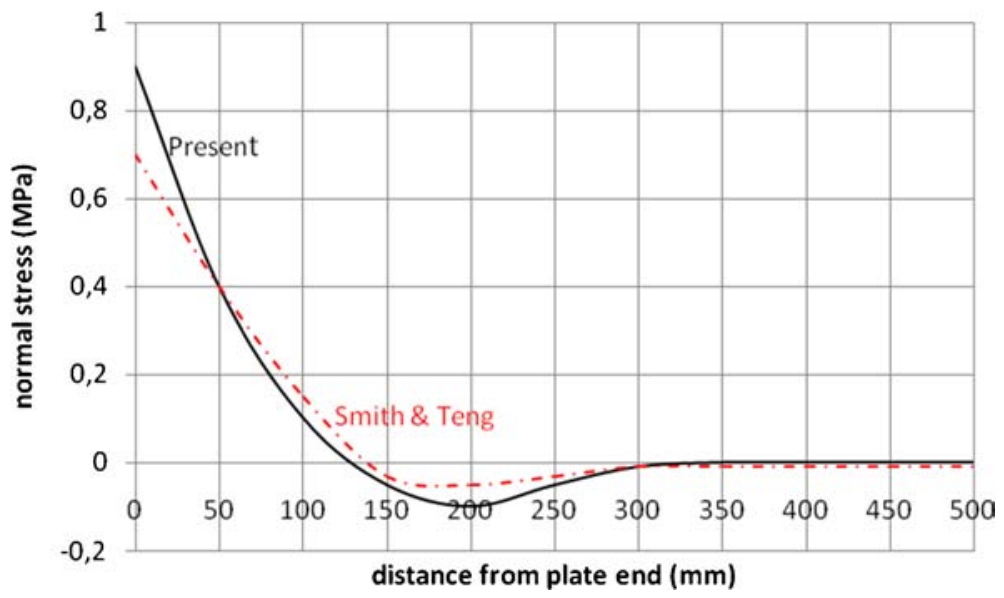

Figure 5. Comparison between the proposed solution and that of Smith and Teng for normal stresses (2001). 


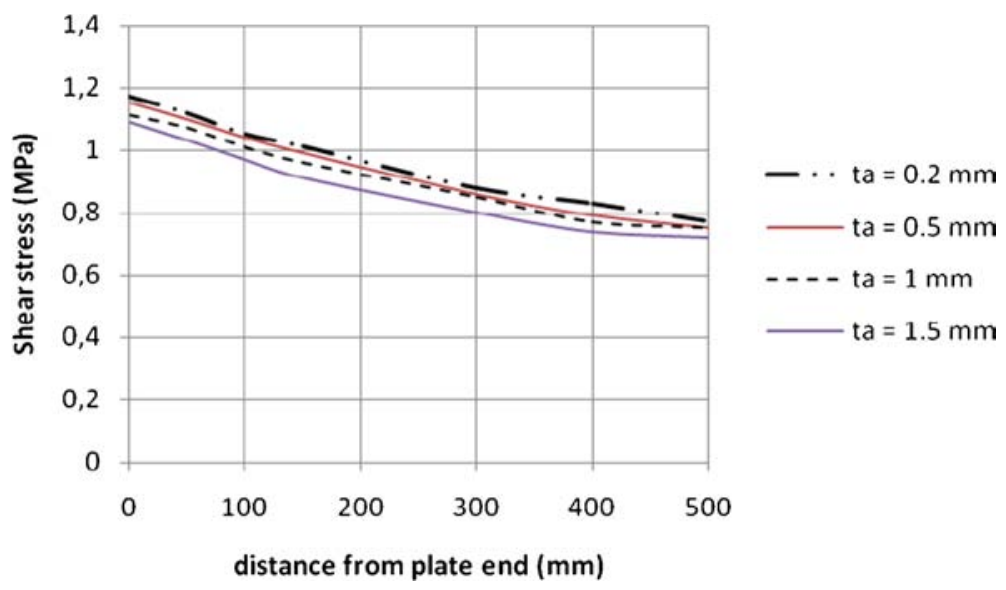

Figure 6. Effect of adhesive layer thickness on interfacial shear stresses.

in both the normal stress and the shear stress, but mostly in shear stress. The normal stresses are particularly affected near the end of the plate. The interfacial stresses increase is due to increased bending moment at the plate end section.

\subsection{Effect of the elastic modulus of adhesive layer}

The adhesive layer is considered to have a small stiffness and is considered as a soft isotropic material. The maximum interfacial stresses are affected by the elastic modulus value. Figure 8 indicates that stiffer adhesive layer produces higher interfacial stresses. They increase approximately linearly as the elastic modulus increases. The predictions of the current study are slightly higher when compared to Smith and Teng's solution.

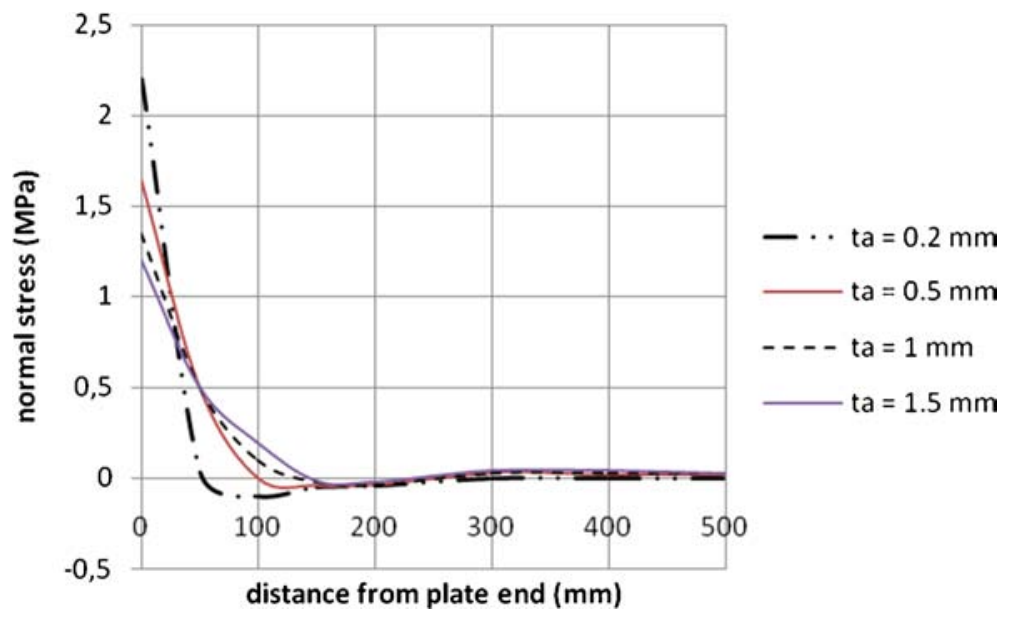

Figure 7. Effect of adhesive layer thickness on interfacial normal stresses. 


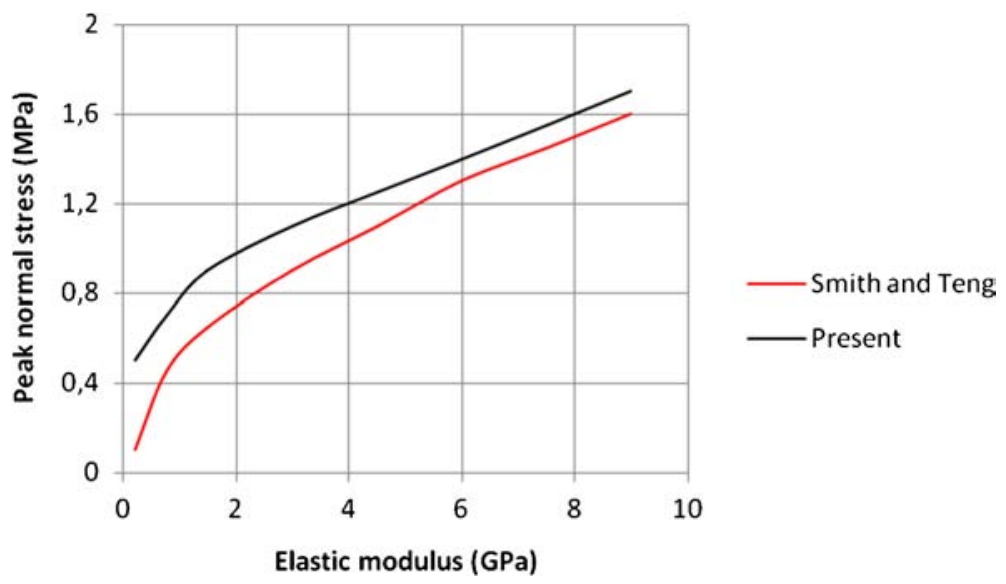

Figure 8. Peak normal stresses vs. elastic modulus of adhesive layer.

\subsection{Effect of FRP plate thickness}

The effect of the FRP plate thickness is shown in figure 9. It can be seen that interfacial stresses increase with increasing the FRP plate thickness. The load is resisted by the FRP plate as its thickness increases. The performance of the analytical solution for the interfacial normal stress slightly improves with an increase in the plate thickness.

Although the distribution of stresses is similar in the model of Smith and Teng in comparison with the present analysis, slight differences near the plate end are observed. The stress distribution in the Smith and Teng model shows sudden drops near the plate ends representing the formation of cracks in these areas.

\subsection{Effect of the elastic modulus of FRP plate}

Different values of elastic modulus of FRP plate are tested. Their effect on the interfacial stresses are shown in figures 10 and 11. It can be seen from these figures that an increase in the elastic

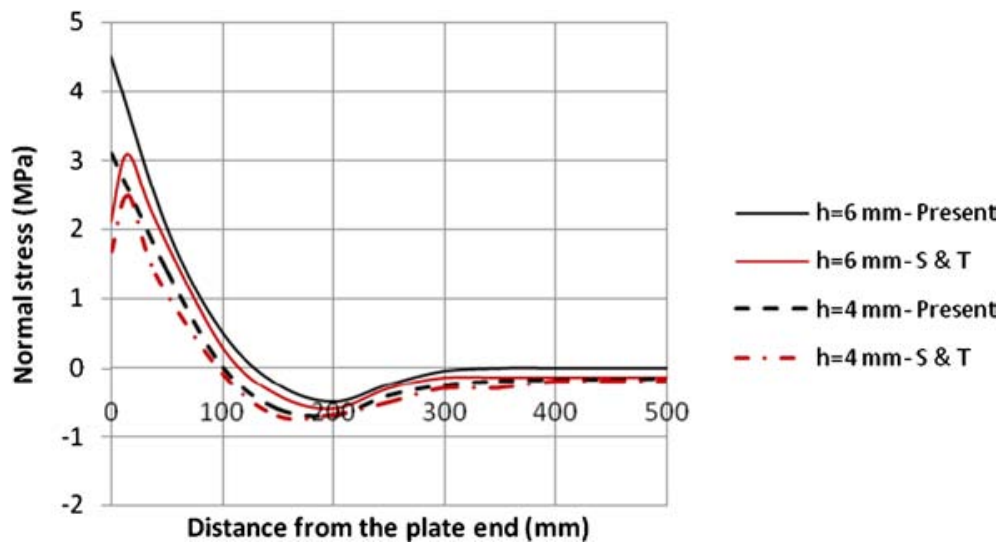

Figure 9. Normal stress vs. FRP plate thickness. 


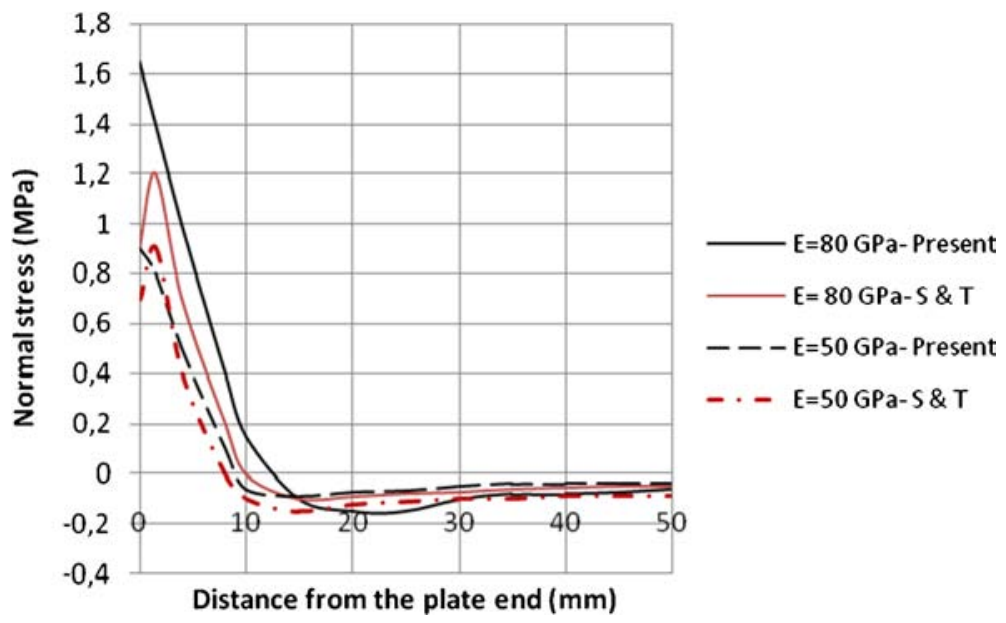

Figure 10. Normal stress vs. elatic modulus of FRP plate.

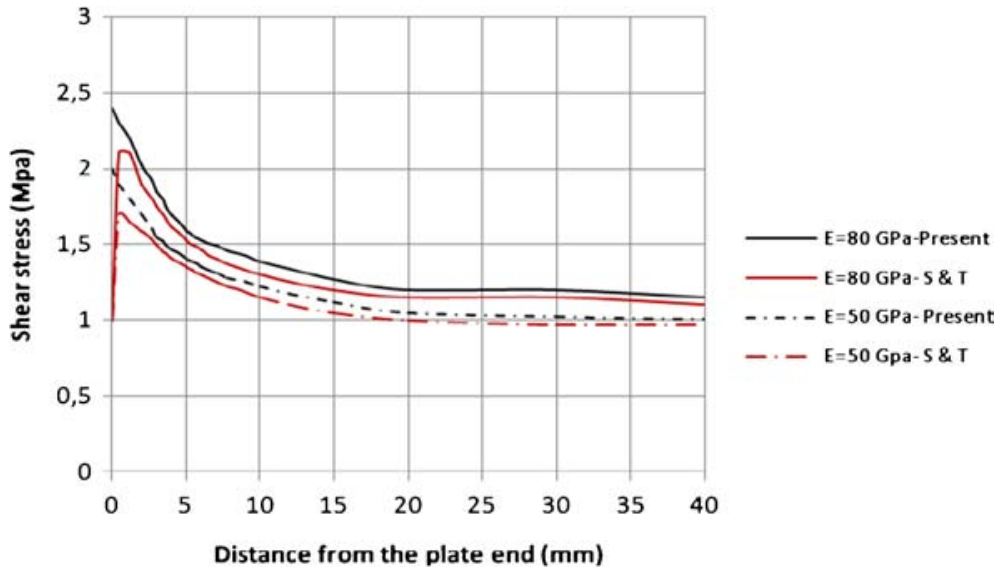

Figure 11. Shear stress vs. elatic modulus of FRP plate.

modulus of FRP plate leads to an increase in the interfacial normal and shear stresses. A stiff plate takes up a larger share of the total load which has to be transferred from the beam to the FRP plate via higher interfacial stresses.

\section{Conclusions}

This paper has presented an analytical solution for interfacial shear and normal stresses in a strengthened beam with a bonded FRP plate by modelling a cohesive zone as a bi-linear bond-slip law. The shear deformation effect in both the beam and the FRP plate are taken into consideration. Different results show a satisfactory agreement between the proposed solution and Smith and Teng solution. The observed effect of shear deformation is not very important. It is noticed that the interfacial stresses are significant near the end of the plate. They decrease 
rapidly to become negligible starting from the first third of the plate. That is also true for the current study and for Smith and Teng' model.

Reducing the thickness of the adhesive layer leads to an increase in both the normal stress and the shear stress, higher interfacial stresses are found for a thinner adhesive layer. Interfacial stresses increase with increasing the FRP plate thickness. This is due to the stiffness of the FRP plate which influences the bending moment. The load is mostly resisted by the FRP as its thickness increases. An increase in the elastic modulus of the FRP plate leads to an increase in the interfacial normal and shear stresses.

Although the distribution of stresses is in general similar in the model of Smith and Teng in comparison with the present analysis, slight differences near the plate end are observed. The stress distribution in the Smith and Teng model shows sudden drops near the plate ends representing the formation of cracks in these areas.

It will be interesting in the future work to look further into the influence of more available cohesive zone models to have a general relationship describing interfacial stresses distribution which will be compared to theoretical and experimental results.

\section{References}

Ahmad O, Gemert D V and Vadewalle L 2001 Improved model for plate-end shear of CFRP strengthened RC beams. Cement Concrete Comp. 23: 3-19

Camacho G and Ortiz M 1996 Computational Modeling of Impact Damage in Brittle Materials. Int. J. Solids and Struct. 33: 2899-2938

Cornetti P and Carpinteri Al 2011 Modelling the FRP-concrete delamination by means of an exponential softening law. Eng. Struct. 33: 1988-2001

Fallah A, Mohamed Ali R and Louca L 2008 Analytical-numerical study of interfacial stresses in plated beams subjected to pulse loading. Eng. Struct. 30: 856-869

Freddi F and Savoia M 2008 Analysis of FRP-concrete debonding via boundary integral equations. Eng. Fract. Mech. 75: 1666-1683

Geubelle P and Baylor J 1998 Impact induced delamination of laminated composites: A 2-d simulations. Composite Part B Eng. 29: 589-602

Hassan T and Rizkalla S 2003 Investigation of bond in concrete structures strengthened with near surface mounted carbon fibre reinforced polymer strips. J. Compos Constr. 7: 248-257

Lorenzis L, Miller B and Nanni A 2001 Bond of fibre-reinforced polymer laminates to concrete. ACI Mater J. 98: 256-264

Lorenzis L and Teng J 2006 Near-surface mounted FRP reinforcement: An emerging technique for strengthening structures. Compos Part B-Eng. 38: 119-143

Lorenzis L and Zavarise G 2009 Cohesive zone modeling of interfacial stresses in plated beams. Int. J. Solids and Structures 46: 4181-4191

Maalej M and Leong K S 2005 Effect of beam size and FRP thickness on interfacial shear stress concentration and failure mode of FRP-strengthened beams. Compos Sci. Technol. 65: 1148-1158

Nakaba K, Kanakubo T, Furuta T and Yoshizawa H 2001 Bond behavior between Fibre-Reinforced Polymer laminates and concrete. ACI Structural J. 98: 359-367

Needleman A 1987 A continuum model for void nucleation by inclusion debonding. J. Appl. Mechanics 54: 525-531

Ortiz M and Suresh S 1993 Statistical properties of residual stresses and intergranular Fracture in Ceramic Materials. J. Appl. Mech. 60: 77-84

Ouyang Z and Wan B 2008 Analytical solution of bi-linear cohesive zone model for FRP bonded concrete specimen under peeling load. Fourth Int. Conf. on FRP Composites in Civil Engineering (CICE2008) 22-24 July 2008, Zurich, Switzerland

Smith S T and Teng J G 2001 Interfacial stresses in plated beams. Eng. Struct. 23: 857-871 
Teng J G, Cheng J F, Smith S T and Lam L 2003 Behavior and strength of FRP-strengthened RC structures: A state-of-the-art review. P I CIVIL ENG-STR B 156: 51-62

Tvergaard V and Hutchinson J W 1993 The Influence of Plasticity on Mixed-Mode Interface Toughness. J. Mechanics and Phys. Solids 41: 1119-1135

Wang J 2007 Cohesive zone model of FRP-concrete interface debonding under mixed-mode loading. Int. J. Solids and Struct. 44(20): 6551-6568

Wu Z and Yin J 2003 Fracture behaviors of FRP-strengthened concrete structures. Eng. Fract. Mech. 70: $1339-1355$

Xu X P and Needleman A 1994 Numerical simulations of fast crack growth in brittle solids. J. Mechanics and Phys. Solids 42: 1397-1434

Yang J, Teng J G and Chen J F 2004 Interfacial stresses in soffit-plated reinforced concrete beams. $P$ I CIVIL ENG-STR B 157: 77-89

Yuan H, Teng J, Seracino R, Wu Z and Yao J 2004 Full-range behavior of FRP-to-concrete bonded joints. Eng. Struct. 26: 553-565 